This is the author's version of an article that has been published in the IEEE Journal of Lightwave Technologies. Changes were made to this version by the publisher prior to publication. The final version of record is available at http://dx.doi.org/10.1109/JLT.2020.3011800

\title{
Coherent Dual-band Radar-over-Fiber Network with VCSEL-based Signal Distribution
}

\author{
Antonio Malacarne, Salvatore Maresca, Filippo Scotti, Paolo Ghelfi, Giovanni Serafino, \\ and Antonella Bogoni, Member, IEEE
}

\begin{abstract}
Today, the advent of radar sensing in applications requiring ever higher precision, such as automotive and industrial monitoring, needs the development of systems with superior resolution, stability and accuracy on 2D/3D spaces. Multiple-input multiple-output (MIMO) radar systems have been studied for more than twenty years, proving to achieve excellent resolutions by merging simultaneous observations from sparse multiple radars. In addition, long-term frequency/phase coherence among distributed signals to and from remote and widely distributed radar frontends, enables a centralized architecture where, in a common base station (BS) site, all the received signals can be coherently processed in synergy, allowing to further improve detection, localization and imaging capabilities. Radio-over-fiber (RoF) solutions represent an enabling technology for guaranteeing coherence among all radar signals coherently generated in the BS and distributed/collected to/from the radar heads by means of optical fiber links. Despite the unprecedented frequency-agility provided by photonics-based radiofrequency (RF) up-/downconversion in the optical domain, for applications requiring massive low-cost production and power efficiency, such as advanced driver-assistance systems, it might be convenient to transmit and receive conventionally generated RF signals, and to limit the use of photonics, as in RoF, to RF signal distribution through cheap high-capacity optical links, in order to avoid EM interference, minimize loss, signal distortion and link encumbrance, still keeping the coherence among distributed signals.
\end{abstract}

In this work, we present a MIMO radar-over-fiber (RaoF) network, where a common BS transmits and receives RF signals through optical standard single-mode fiber (SSMF), to and from two remote radar transmitters (TXs) and four receivers (RXs), all remote with respect to the $B S$ and distributed on a $3 \mathrm{~m}$-long baseline, and employing cheap patch antennas. Electro-optic conversion is achieved through direct modulation (DM) of lowcost, power-effective and high-speed vertical-cavity surfaceemitting lasers (VCSELs) working in the $1.3 \mu \mathrm{m}$-wavelength regime. Such a wavelength ensures propagation over SSMF with negligible distortions up to the km-range. The system is tested in a down-scaled indoor scenario and an RF carrier of $8.5 \mathrm{GHz}$ is used, due to the limited frequency response of the employed VCSELs. System performance is evaluated with $1.4 \mathrm{GHz}$-bandwidth signals, comparing non-coherent and coherent signal elaboration. In addition, dual-band operation in the same RF region is tested,

This paragraph of the first footnote will contain the date on which you submitted your paper for review. It will also contain support information, including sponsor and financial support acknowledgment. For example, "This work was supported in part by the U.S. Department of Commerce under Grant BS123456."

The next few paragraphs should contain the authors' current affiliations, including current address and e-mail. For example, F. A. Author is with the confirming that VCSEL-based optical links are a viable way to guarantee phase coherence among the distributed signals and among multiple frequency bands. Coherent MIMO processing also confirm the potential to achieve superior range and crossrange resolution than non-coherent data fusion.

Index Terms - MIMO radar, Multi-band radar, Optical fiber, Radar over fiber, Radar signal processing, Ultra-wideband radar, Vertical cavity surface emitting laser, automotive.

\section{INTRODUCTION}

$\mathrm{R}$ ADAR systems, whose invention dates back to well over a hundred years ago, have continuously expanded their application areas throughout their history. Over time, human beings have increasingly relied on radar sensors: historical applications such as air and naval traffic monitoring, have been added over time to the most disparate scenarios: on-board systems supporting aerial driving, monitoring of atmospheric conditions, deforestation and pollution factors through satellite observations, evaluation of landslide risks, surveillance of hydrogeological phenomena, just to name a few. The massive use of radar is mainly due to its ability to operate continuously in all weather conditions, making it extremely reliable. Indeed, performance of other types of sensors such as high-precision and infrared cameras or light detection and ranging (LIDAR) systems, degrades with weather conditions and exhibits much stronger limitations, especially in terms of operation distance. The ever increasing miniaturization of electronic components also promoted a progressively more pervasive use of radars up to the latest high-precision applications, such as road traffic monitoring, collision avoidance systems, control of industrial assembly lines and conveyors and body-scan imaging for medicine and security [1],[2]. Thus, to meet constantly higher demands imposed by those applications in terms of resolution, stability and accuracy within a coverage volume, radars have started becoming even more ubiquitous sensors. [3].

Although, by using large bandwidth, quite a high range resolution can be reached, angular or cross range resolution is intrinsically limited by the antenna geometry. This can be improved by rotating radars and even more advanced ones

National Institute of Standards and Technology, Boulder, CO 80305 USA (email: author@boulder.nist.gov).

S. B. Author, Jr., was with Rice University, Houston, TX 77005 USA. He is now with the Department of Physics, Colorado State University, Fort Collins, CO 80523 USA (e-mail: author@lamar.colostate.edu).

T. C. Author is with the Electrical Engineering Department, University of Colorado, Boulder, CO 80309 USA, on leave from the National Research Institute for Metals, Tsukuba, Japan (e-mail: author@nrim.go.jp). 
$>$ REPLACE THIS LINE WITH YOUR PAPER IDENTIFICATION NUMBER (DOUBLE-CLICK HERE TO EDIT) <

This is the author's version of an article that has been published in the IEEE Journal of Lightwave Technologies. Changes were made to this

version by the publisher prior to publication. The final version of record is available at http://dx.doi.org/10.1109/JLT.2020.3011800

based on active electronically scanned array and phased arrays for beamforming operation, and a further improvement is possible with the synthetic aperture radar (SAR) approach [4], that consists in observing the same scene from different points of view by using a moving radar. Since, in many of the abovementioned scenarios, it is not feasible to continuously scan the radar position as it happens in satellite observations, an alternative relies in realizing networks of spatially distributed radars. Such a system is capable of simultaneously observing the scene from different viewpoints, leading to evident benefits in the accuracy of target detections, especially of those with high angular radar cross section (RCS) variability, such as low observable targets, e.g. stealth targets in military environments.

In a recent class of radar systems, named multiple-input multiple-output (MIMO), a multitude of radar nodes operate in synergy, in such a way multiple transmission waveforms are received by multiple receivers enabling jointly elaboration of the collected data [5]-[7]. By exploiting the processed information collected from different spatial positions, an excellent cross-range resolution can be achieved, which is basically independent of the single antenna characteristics. In addition, if all the received signals are collected at a base station common to all the sensors and a mutual coherence among them is guaranteed, a centralized elaboration of raw data, rather than pre-elaborated ones, theoretically leads to a further improvement of detection and localization capabilities [8]-[11]. Generic multistatic radar systems employ multiple transmitting and receiving radar nodes that might be closely and/or widely separated. Widely separated antennas, in the generic case of anisotropic targets, lead to collect a larger percentage of the power backscattered by the target, for handling slow-moving targets, by exploiting Doppler estimates from different aspect angles, and for granting high-resolution target localization. However, such a wide distribution of coherent sensors over a baseline -equivalent to TX and RX phased arrays with sparse elements implementing beamforming functionalities - also causes the rise of sidelobes for each main lobe corresponding to a detected target [8]. This may easily lead to undesirable false alarms and location errors. Some methods for optimizing the sensor distribution geometry in case of wide separation, so as to minimize the sidelobe contribution with respect to the main lobe one, i.e. sidelobe suppression ratio, are recently being proposed [12]. This way, a tradeoff between system complexity/cost and capabilities can be identified.

On the other hand, an improvement of the range resolution is also desirable, which is theoretically imposed by the investigated RF bandwidth. In addition, radars transmitting multiple coherent bands may provide additional information to enhance target classification and detection by means of improved classification algorithms [13]-[15], enable multispectral imaging of complex targets [16] and estimate environment scattering characteristics [17],[18]. Another motivation pushing multi-band solutions is that spectral erosion driven by the ever-expanding wireless communication services leaves only sparse and narrow bands available for radar sensing. Furthermore, given the use of band-limited RF components, a radar system based on multiple bandwidth-limited frontends, each one operating at a distinct frequency, may be preferable with respect to a single ultrawide-band frontend [15],[18]. In addition, a multi-band architecture with intrinsic coherence among bands allows synthetization of larger bands through the so-called spectral gap filling algorithms avoiding, at the same time, frequency and phase recovery strategies [19]-[22].

Merging the advantages of a coherent MIMO system with those enabled by multi-band operation, may lead to a new class of radar systems with unique features. However, coherent distribution along a sparse radar network of RF signals at different frequency carriers is challenging. Recently, photonics has proved to be extremely effective in guaranteeing coherent multi-band signal distribution [18],[23]. Some recent works have demonstrated that direct modulation (DM) of laser diodes - in particular of vertical-cavity surface emitting lasers (VCSELs) - represents an attractive low-cost, power-efficient and robust solution for coherent distribution of analog RF signals [24],[25]. DM-VCSELs offer a higher RF-to-optical power conversion than other options such as distributed feedback (DFB) lasers, and their shorter cavity potentially leads to faster modulation response which goes well with the everincreasing frequencies used by the new generation radars. In addition, their robustness to high temperature allows uncooled operation [26]-[28]. Concerning the choice on their emission wavelength, the general trend is that shorter-wavelength VCSELs are cheaper, reach higher modulation speed (> $30 \mathrm{GHz}$ at $850 \mathrm{~nm}$, [29]) and emission power, but exhibit higher losses and signal distortion due to chromatic dispersion (CD) and multi-mode transmission, whilst longer ones present narrower modulation bandwidth approaching $20 \mathrm{GHz}$ [30] with minimized distortions. A solution to achieve coherent signal distribution with minimum distortions at ultra-high carrier frequency frontends ( $\geq 40 \mathrm{GHz}$ ) up to km-range fiber reach, is to employ $1.3 \mu \mathrm{m}$-VCSELs (single-mode transmission and minimum CD) with radar heads like the ones presented in [31], where integrated $80 \mathrm{GHz}$-radars include internal $\times 4$ and $\div 4$ frequency multiplier and divider, respectively.

The main objective of the article is to demonstrate that, thanks to the coherence provided by VCSEL-based RF signal distribution, coherent multiband MIMO processing is successfully applied to a Radar-over-Fiber (RaoF) network, where remoting of transmitters (TXs) and receivers (RXs) with respect to a centralized base station is achieved through DM1.3 $\mu \mathrm{m}$-VCSELs [24],[25]. However, due to the speed limitations imposed by the employed VCSELs, 8.5 GHz-carrier frequency of the distributed RF signals was chosen.

The remainder of this manuscript is organized as follows. Section II introduces the concept and modeling of a MIMO radar system. In Section III the introduced non-coherent and coherent MIMO processing is successfully applied to an actual VCSEL-based photonics MIMO radar system. Section IV presents the potentials of a dual-band approach using the same hardware. Conclusions and discussions are finally exposed.

\section{MIMO RADAR NETWORK}

The general case of a MIMO radar system observing multiple targets (point-like scattering sources) using different signals on 
$>$ REPLACE THIS LINE WITH YOUR PAPER IDENTIFICATION NUMBER (DOUBLE-CLICK HERE TO EDIT) <

This is the author's version of an article that has been published in the IEEE Journal of Lightwave Technologies. Changes were made to this version by the publisher prior to publication. The final version of record is available at http://dx.doi.org/10.1109/JLT.2020.3011800 multiple frequency bands, is described in this Section. Some modeling assumptions are done to facilitate the study.

\section{A. Multi-Target Multi-Band Signal Model}

Let us consider a MIMO radar system composed by $M$ TXs and $N$ RXs, which contemporaneously illuminate $K$ point-like scattering elements, belonging to one single target or to multiple targets, in the monitored area. Moreover, let us assume that each transmitter can transmit on $L$ different carrier frequencies. With no loss of generality, we can consider $s_{m}(t)$ being the low-pass equivalent of all the $L$ signals transmitted by the $m^{\text {th }}$ TX. Thus, the generic signal $r_{m, n, l}(t)$, which is received by the $n^{\text {th }} \mathrm{RX}$, can be written as [8]:

$$
r_{m, n, l}(t)=\sum_{k=1}^{K} a_{m, n, l}^{(k)} S_{m}\left(t-\tau_{m, n}^{(k)}\right) e^{j \varphi_{l}(t)}+w_{n}(t)
$$

where $a_{m, n, l}^{(k)}(t)$ and $\tau_{m, n}^{(k)}$ are, respectively, the complex amplitude and delay characterizing the $\mathrm{k}^{\text {th }}$ scattering element, with $m=1, \ldots, M, n=1, \ldots, N \quad$ and $l=1, \ldots, L$. Both parameters are functions of the bistatic geometry between the scatterer location $\left(x_{k}, y_{k}\right)$ and the sensors position in the 2D Cartesian plane $(x, y)$. However, for simplicity only the amplitude coefficients are function of the $l^{\text {th }}$ employed carrier. In fact, we assume that the same $K$ point-like scatterers are observed by all the TXs and RXs at the $L$ employed carrier frequencies. In the following, for brevity, we will omit the dependence of $a_{m, n, l}^{(k)}(t)$ and $\tau_{m, n}^{(k)} \tau_{\mathrm{m}, \mathrm{n}}^{(\mathrm{k})}$ on $\left(x_{k}, y_{k}\right)$, while the amplitude factor will be assumed as an unknown deterministic constant. The term $w_{m}(t)$ is modelled as an additive white Gaussian noise (AWGN) stochastic process, while the terms $\varphi_{l}(t)$ takes into account the phase shift caused by the optical oscillator instability at the $L$ employed carrier frequencies. For simplicity, no bistatic clutter contribution is considered.

\section{B. MIMO Data Fusion Concept}

To estimate the target position, the maximum of the likelihood function of the target location $\vec{X} \equiv(x, y)$ must be evaluated from the set of all the $M N L$ available received signals $r_{m, n, l}(t)$. Thus, the target position can be estimated as the location $\vec{X}$ for which the log-likelihood function, namely the MIMO ambiguity function $A(\vec{X})$, reaches its maximum. As described in [8], there are two ways to calculate the MIMO ambiguity function, which suggest two complementary functionalities named "searching" and "imaging" modes. As described in [32], the searching mode is addressed by calculating the "non-coherent" MIMO ambiguity function [8]:

$$
A_{N C}(\vec{X}) \propto \sum_{m=1}^{M} \sum_{n=1}^{N} \sum_{l=1}^{L}\left|\Psi_{m, n, l}\left(t, \tau_{m, n}\right)\right|^{2}
$$

where:

$$
\Psi_{m, n, l}\left(t, \tau_{m, n}\right) \triangleq \int r_{m, n, l}^{*}(t) s_{m}\left(t-\tau_{m, n}\right) d t
$$

According to Eq. (2), for each possible target location $(x, y)$ in the Cartesian search space, the ambiguity function is computed by determining the $M N L$ cross-correlations between the received and transmitted base-band equivalent signals, see Eq. (3), where $*$ indicates the operation of complex conjugation. Conversely, the imaging mode is addressed by calculating the "coherent" MIMO ambiguity function:

$$
A_{C}(\vec{X}) \propto\left|\sum_{m=1}^{M} \sum_{n=1}^{N} \sum_{l=1}^{L} e^{-j 2 \pi f_{R F}^{(l)} \tau_{m, n}} \Psi_{m, n, l}\left(t, \tau_{m, n}\right)\right|^{2}
$$

In the exponential terms $e^{-j 2 \pi f_{R F}^{(l)} \tau_{m, n}}$, which depend on the $l^{\text {th }}$ carrier frequency $f_{R F}^{(l)}$ with $l=1, \ldots, L$, each $\tau_{m, n}$ takes into account both the 2D Euclidean distances from the $m^{\text {th }}$ transmitter to the generic target location $\vec{X}$ and the one from $\vec{X}$ to the $n^{\text {th }}$ receiver. Those exponential terms are therefore used to re-align the phases of the signals such that the complex correlation contributes can be summed together coherently. As underlined in [32], if the angular jitter of the whole system is smaller than 0.1rad, Eq. (4) can be successfully applied.

\section{VCSEL-BASED MIMO RADAR NETWORK EXPERIMENTS}

A down-scaled indoor experimental setup has been realized to demonstrate the operational capabilities of a MIMO radarover-fiber system based on the hardware of the TX and RX presented in [24], see Fig. 1. A base station (BS) is responsible for delivering the RF signals to two remote TXs and collects the RF echo signals from four remote RXs, through SSMF spools approximately $10 \mathrm{~m}$-long each, such that a $2 \times 4$ MIMO system emulating eight separate channels is realized. Electro-optical conversion of the generated and received RF signals is achieved through the use of VCSELs, whereas opto-electrical conversion takes place through photodiodes. Two channels of a Fujitsu digital-to-analog converter (DAC) with sampling rate equal to $60 \mathrm{GS} / \mathrm{sec}$ (with a memory space of $2048 \times 128$ samples) are used to generate repetitive 273 ns-long linearly frequencymodulated continuous-wave (LFM-CW) signals with $1.4 \mathrm{GHz}-$ bandwidth and a central frequency of $8.5 \mathrm{GHz}$. The choice of such a frequency is due to the limited $3 \mathrm{~dB}$-bandwidth provided by the employed solution (see [24]). $\mathrm{TX}_{1}$ and $\mathrm{TX}_{2}$ transmit upand down-sweep waveforms, respectively. In addition, the two signals have been separated in the time domain too, to

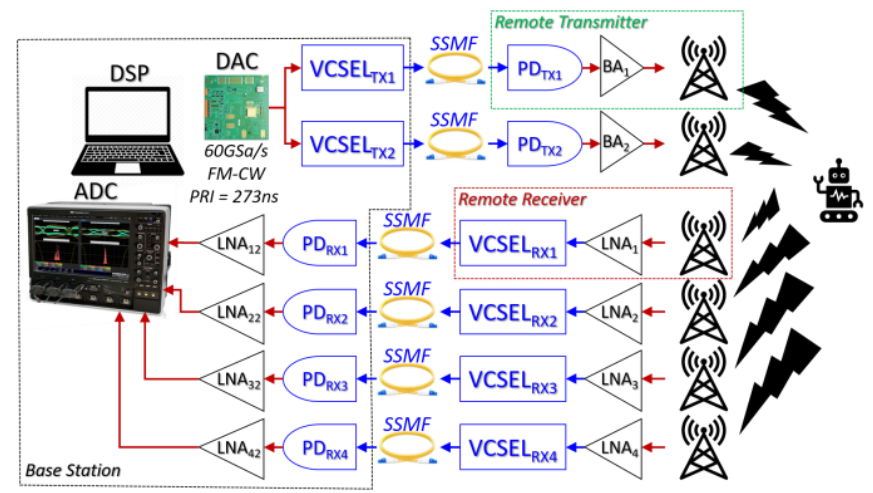

Fig. 1 Experimental setup implementing the $2 \times 4$ VCSEL-based MIMO radar network 
This is the author's version of an article that has been published in the IEEE Journal of Lightwave Technologies. Changes were made to this verșion by the publisher prior to publication. The final version of record is available at http://dx.doi.org/10.1109/JLT.2020.3011800

maximize signal orthogonality. The two waveforms are interleaved so that each TX is active for one waveform repetition interval and is silent for the following interval, when it is the turn of the other TX to send its signal. The number of RXs has been limited to four because of the maximum number of channels available on the employed real-time oscilloscope employed as ADC (Teledyne LeCroy SDA-813Zi-A, 40 GS/s on each of the four channels, $13 \mathrm{GHz}$-analog bandwidth). A third DAC channel was used to trigger the ADC (oscilloscope) and both DAC and ADC were referenced with a common $10 \mathrm{MHz}$ tone. The BS (see Fig. 1) includes electro-optical conversion of the LFM-CW signals through direct modulation of two VCSELs, and acquisition of the four RXs outputs through $\mathrm{ADC}$, after photodetection $\left(\mathrm{PD}_{\mathrm{RX} 1.4}\right)$ and low-noise amplification (LNA). All the hardware components are replicas of what reported in [24] except for the TX antennas which have been substituted with Vivaldi-shaped wideband horn antennas with about $12 \mathrm{dBi}$ of maximum gain.

Each TX also includes a photodiode ( $\mathrm{PD}_{\mathrm{TX} 1,2}$ ) followed by a booster RF amplifier $\left(\mathrm{BA}_{1,2}\right)$. In each $\mathrm{RX}$ a custom patch antenna $(6.56 \mathrm{~cm} \times 8.67 \mathrm{~cm})$, designed on Rogers $4350 \mathrm{~b}$ to exhibit a gain of $6 \mathrm{dBi}$ in the frequency range $6-11 \mathrm{GHz}$ for a $120^{\circ}$-field of view, feeds a $40 \mathrm{~dB}$-gain RF low-noise electrical amplifier (LNA) that properly amplifies the received signal to modulate the VCSEL. Additional hardware details can be found in [24].

As reported in Fig. 2, the antennas are placed on a 1D 3mlong baseline, thus enabling 2D imaging ( $x-y$ plane). The exact location of each antenna is included in the figure. As a target, a cylinder can with a radar cross section (RCS) of $-7 \mathrm{dBsm}$ (assumed omnidirectional on the range/cross-range plane with position coordinates $(0,+3.6) \mathrm{m}$, was used.

The signals acquired via the four channels of the

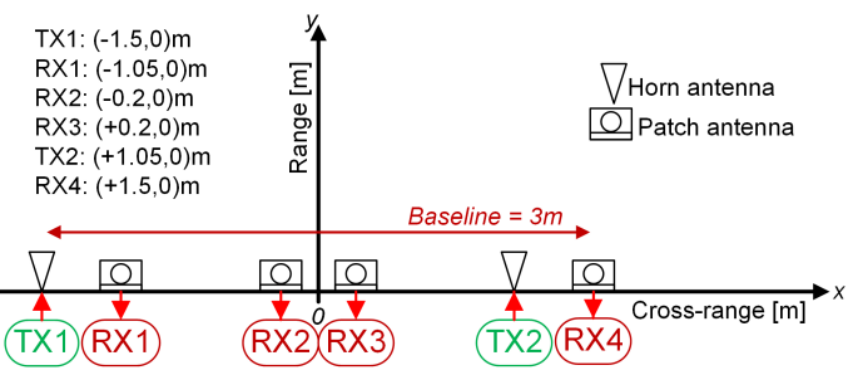

Fig. 2 Disposition of antennas along the baseline for the MIMO radar network demonstration.

oscilloscope, are processed as follows. First, the four received signals are down-converted from RF to baseband (BB). Then, the BB-equivalent signals, which can be described by Eq. (1) with $M=2, N=4$ and $L=1$ (i.e., single-band operation), are cross-correlated with the two reference waveforms (i.e., the ones employed at the two TXs), thus obtaining $M N L=8$ vectors, one for each $\mathrm{TX} / \mathrm{RX}$ virtual channel. It is worth noticing that, in a pre-calibration process, the delay of each $\mathrm{TX} / \mathrm{RX}$ virtual channel is compensated for length differences of fibers, cables and circuitry. In addition, the amplitude response mismatch among different virtual channels, which is due to the different behavior of distinct VCSELs and electrical amplifiers, is compensated in post-processing.. To this aim, both delays
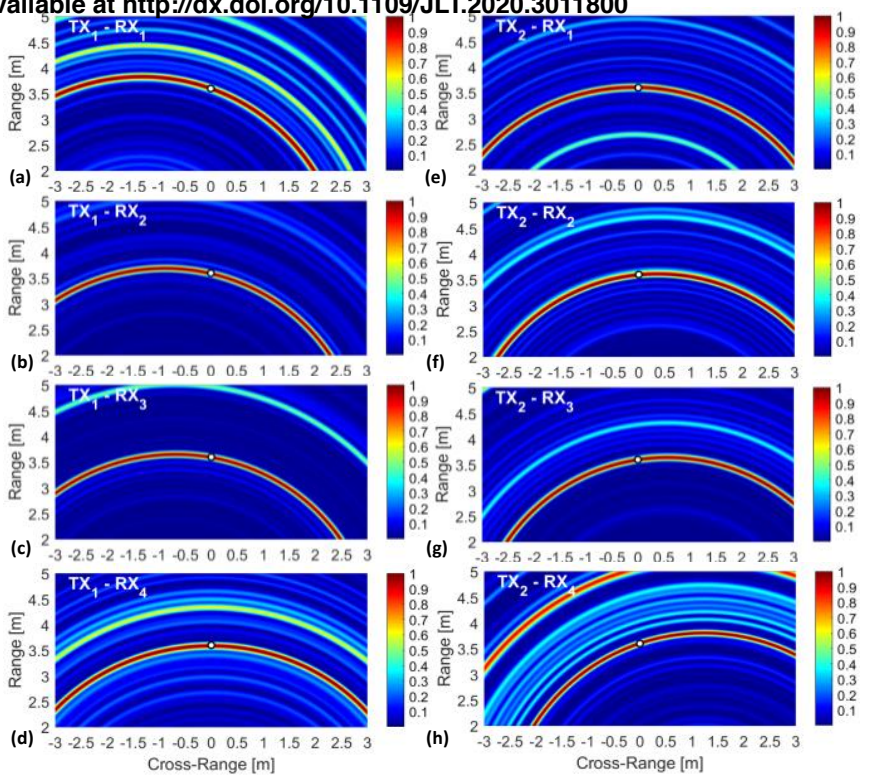

Fig. 3 Single-band 2x4 MIMO radar experiment. Color-coded normalized squared modulus of the cross-correlation vector calculated at each bistatic radar channel after 2D mapping: (a) $\mathrm{TX}_{1}-\mathrm{RX}_{1}$, (b) $\mathrm{TX}_{1}-\mathrm{RX}_{2}$, (c) $\mathrm{TX}_{1}-\mathrm{RX}_{3}$, (d) $\mathrm{TX}_{1}-\mathrm{RX}_{4}$, (e) $\mathrm{TX}_{2}-\mathrm{RX}_{1}$, (f) $\mathrm{TX}_{2}-\mathrm{RX}_{2}$, (g) $\mathrm{TX}_{2}-\mathrm{RX}_{3}$, (h) $\mathrm{TX}_{2}-\mathrm{RX}_{4}$. Experimental parameters: $f_{R F}=8.5 \mathrm{GHz}, B=1.4 \mathrm{GHz}$; Target position $T \equiv$ $(0,+3.6)$ m denoted by the white dot.

and amplitudes are measured for all the combinations of TXs and RXs, in a back-to-back configuration.

At this point, the complex cross-correlation vectors $\Psi_{m, n, l}\left(t, \tau_{m, n}\right)$ are evaluated for all the $m, n, l$ radar channel combinations, with $m=1, \ldots, M, n=1, \ldots, N$ and $l=1, \ldots, L$, following Eq. (3). Since they are functions of the TX-target-RX travelled time $\tau_{m, n}$, which is in turn proportional to the bistatic range, the cross-correlation vectors can be mapped via geometric transformation into complex bi-dimensional (2D) matrices. In fact, given the position of the generic $m^{\text {th }} \mathrm{TX}$ and $n^{\text {th }} \mathrm{RX}$ element, which act as the two foci, the peaks displayed within the cross-correlation vector transform into ellipsoidal wave fronts by varying the range $(y)$ and the cross-range $(x)$, as depicted in Fig. 3.

Finally, both non-coherent and coherent MIMO processing are performed for comparison, by means of Eq. (2) and Eq. (4), respectively. A possible use of such a system is to continuously perform non-coherent processing on the coverage volume for target detection and, around the detected ones, apply finer coherent processing for target identification purposes. In the first output type, the amplitudes of the eights complex channel matrices are summed (i.e., non-coherent summation). In the second output type, the complex channel matrices are first multiplied by a further "phase-alignment" matrix, which compensates for the phase shifts due to the different signal travel delays in free space. Finally, the resulting phase-aligned matrices are summed together, giving a single matrix representing the observed scenario. The MIMO radar processing tools have been developed through Matlab codes running offline, after data acquisition, on a laptop.

\section{A. Non-coherent MIMO processing}

In order to assess the 2D imaging capability of the proposed photonics-based radar network, as a first step the system has 
This is the author's version of an article that has been published in the IEEE Journal of Lightwave Technologies. Changes were made to this version by the publisher prior to publication. The final version of record is available at http://dx.doi.org/10.1109/JLT.2020.3011800 been configured to perform non-coherent MIMO processing. The single radar detections (i.e., the amplitudes of the crosscorrelation matrices after 2D mapping) of each bistatic TX/RX pair are considered in the subplots of Fig. 3. As we can observe, in each one of the eight insets, the target (white dot) is clearly detected by the red ellipsoid curves above the background clutter. This latter, appearing as multiple color-coded ellipsoids unpredictably distributed in each of the insets, could be originated by multiple propagation modes from the room walls, clutter due to furniture or from the target itself.

As the TX/RX bistatic radar pairs do not provide any angular information (the employed RX antennas are almost omnidirectional), target likelihood functions are spread over bistatic iso-range ellipsoids whose foci, as already said, correspond to the generic TX and RX positions. Finally, the non-coherent MIMO output, which is displayed in Fig. 4(a), consists of the super-position of the eight bistatic detection maps. By observing the outputs, the range resolution is confirmed to be $12 \mathrm{~cm}$, according to the employed $1.4 \mathrm{GHz}$ bandwidth radar waveform. Conversely, the cross-range resolution is $62 \mathrm{~cm}$ and it depends on the geometry of the radar array with respect to the target position. For the sake of conciseness, only the main performance metrics are reported in
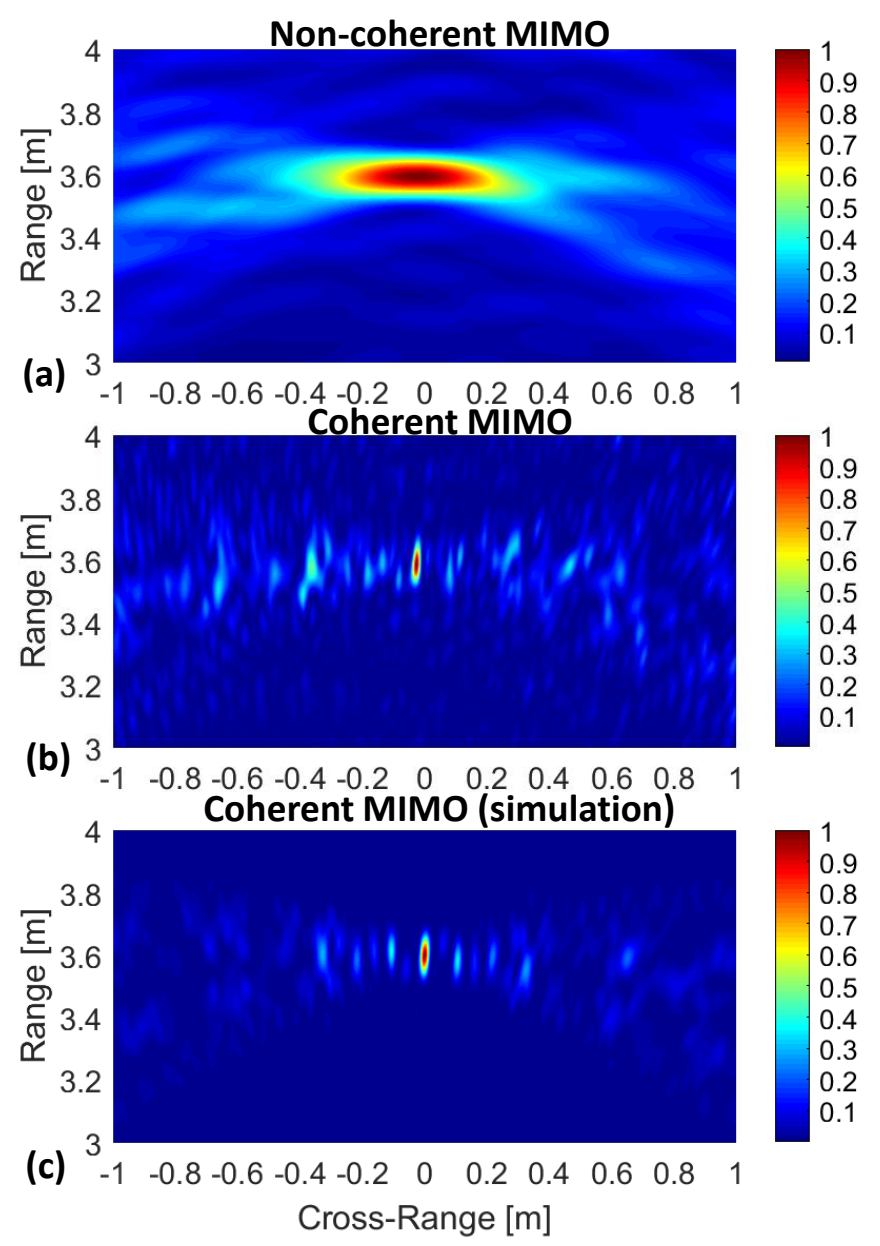

Fig. 4 Single-band 2x4 MIMO radar experiment. Color-coded normalized MIMO ambiguity function in the range/cross-range Cartesian space for: (a) non-coherent MIMO processing of real data, (b) coherent MIMO processing of real data, (c) coherent MIMO processing of simulated data. Experimental parameters: $f_{R F}=8.5 \mathrm{GHz}, B=1.4 \mathrm{GHz}$; Target position $T \equiv(0,+3.6) \mathrm{m}$.

\begin{tabular}{ccccc}
\multicolumn{4}{c}{ PERFORMANCE METRICS FOR THE SINGLE-BAND EXPERIMENT } \\
\hline \multicolumn{4}{c}{ Simulated Data } & \multicolumn{2}{c}{ Experimental Data } \\
& NC-MIMO & C-MIMO & NC-MIMO & C-MIMO \\
\hline$\Delta R$ & $0.115 \mathrm{~m}$ & $0.095 \mathrm{~m}$ & $0.12 \mathrm{~m}$ & $0.105 \mathrm{~m}$ \\
$\Delta X R$ & $0.565 \mathrm{~m}$ & $0.025 \mathrm{~m}$ & $0.62 \mathrm{~m}$ & $0.025 \mathrm{~m}$ \\
PMSR & - & $4.03 \mathrm{~dB}$ & - & $4.31 \mathrm{~dB}$ \\
PASR & - & $15.01 \mathrm{~dB}$ & - & $8.13 \mathrm{~dB}$ \\
\hline
\end{tabular}

Sidelobe statistics cannot be evaluated in the case of non-coherent MIMO. NC-MIMO: Non-Coherent MIMO; C-MIMO: Coherent MIMO; $\Delta R$ : Range Resolution; $\triangle X R$ : Cross-Range Resolution; PMSR: Peak-to-MaximumSidelobe-Ratio; PASR: Peak-to-Average-Sidelobe-Ratio.

Table I (columns 1 and 3), demonstrating a good agreement between the simulation model, which considers a point-like scattering target, and the real experimental conditions. As can be easily foreseen, the cross-range resolution achieved through non-coherent processing considerably limits the system for closely-spaced targets, as already pointed out in [33].

\section{B. Coherent MIMO processing}

This paragraph exposes how frequency/phase coherence between the transmitted and received RF signals considerably impacts on the potential performance of the conceived VCSELbased RaoF MIMO network.

To apply coherent MIMO processing (see Eq. 4), the exponential term $e^{-j 2 \pi f_{R F}^{(l)} \tau_{m, n}}$ is numerically calculated for each $(x, y)$ position of the 2D map and for each TX/RX pair, so as to obtain the $2 \times 4=8$ phase-alignment matrixes.

As mentioned in the introduction, the coherent MIMO ambiguity function, see eq. (4), can be considered equivalent to a beamforming operation applied to a sparse array of antennas. However, due to the antenna sparseness, unwanted sidelobes may appear in the final array beampattern, as shown in Fig. 4(b),(c). Their number, intensity and position depend on the system parameters (e.g., sensors-target geometry, carrier frequency, etc.). In order to better evaluate the correct functioning of the system, a way to compare sidelobes with the peak originated by the target, is fundamental. For this reason, two metrics can be defined for quantifying the maximum and average intensity level of the sidelobes with respect to the main lobe. These metrics are the peak-to-maximum sidelobe ratio (PMSR) and peak-to-average sidelobe ratio (PASR), which are respectively calculated as:

$$
\begin{aligned}
& \mathrm{PMSR} \triangleq \max _{\bar{k} \in K}\left\{A_{C}^{(\bar{k})}\right\} / \max _{k \in K \backslash \bar{k}}\left\{A_{C}^{(k)}\right\}, \\
& \mathrm{PASR} \triangleq \max _{\bar{k} \in K}\left\{A_{C}^{(\bar{k})}\right\} / \operatorname{mean}_{k \in K \backslash \bar{k}}\left\{A_{C}^{(k)}\right\},
\end{aligned}
$$

where $A_{C}^{(k)}$ is the value of the coherent ambiguity function at the $k^{\text {th }}$ local maximum, $\bar{k}$ is the index of the absolute maximum corresponding to the position of the target, while $K$ represents the total number of local maxima of $A_{C}(\vec{X})$. In addition to the aforementioned PMSR and PASR metrics, the range and crossrange resolutions, respectively denoted by $\Delta R$ and $\Delta X R$, will be estimated from the MIMO ambiguity functions, by considering the width of the main lobe at $-3 \mathrm{~dB}$.

The result of coherent MIMO processing is shown in Fig. 4(b), where a strong improvement in cross-range resolution 
This is the author's version of an article that has been published in the IEEE Journal of Lightwave Technologies. Changes were made to this version by the publisher prior to publication. The final version of record is available at http://dx.doi.org/10.1109/JLT.2020.3011800

with respect to the non-coherent approach (see Fig. 4(a)), is clear. As a matter of fact, while in a single-band radar system the range resolution, which is determined by the signal bandwidth, remains almost unchanged, the angular resolution can be calculated as the ratio between the wavelength of the RF carrier and the baseline length. In this case, being the wavelength $3.5 \mathrm{~cm}$ and the baseline $3 \mathrm{~m}$, the theoretical crossrange resolution at $3.6 \mathrm{~m}$ range is about $2.6 \mathrm{~cm}$, in excellent agreement with the values reported in Table I (columns 2 and 4, namely $2.7 \mathrm{~cm}$ for the experiment, $2.6 \mathrm{~cm}$ for simulation). However, by observing the plot, beside the peak corresponding to the target, other secondary peaks are clearly visible, leading to a PASR of $8.1 \mathrm{~dB}$. Their distribution in correspondence of each main lobe is due to the abovementioned array element beampattern which depends on the limited number of TXs and RXs and, therefore, the number of MIMO channels.

For completeness, the same scenario has been simulated considering an ideal point-scatterer located at the same target position as in the indoor experiment. The output is shown in Fig. 4(c) to verify up to which extent the results from the real and simulated data are similar. As we can observe from the comparison between Fig. 4(b) and Fig. 4(c), the two plots are almost identical, confirming the position and the amplitude of the secondary lobes. The difference between the two results shall be ascribed to three main reasons: $i$ ) the scattering nature of the target, which is not a point-like scatterer, but rather a distributed target, ii) the unpredictable multipath scattering features of the background in the indoor experiment, iii) the limited temporal granularity given by the ADC sampling rate $40 \mathrm{GS} / \mathrm{s}$ - which leads to approximately a phase accuracy of $2 \pi / 5$ when estimating and then applying the mentioned phasealignment matrixes, $i v)$ unpredictable temperature-dependent variations of the VCSEL gain which are not compensated and

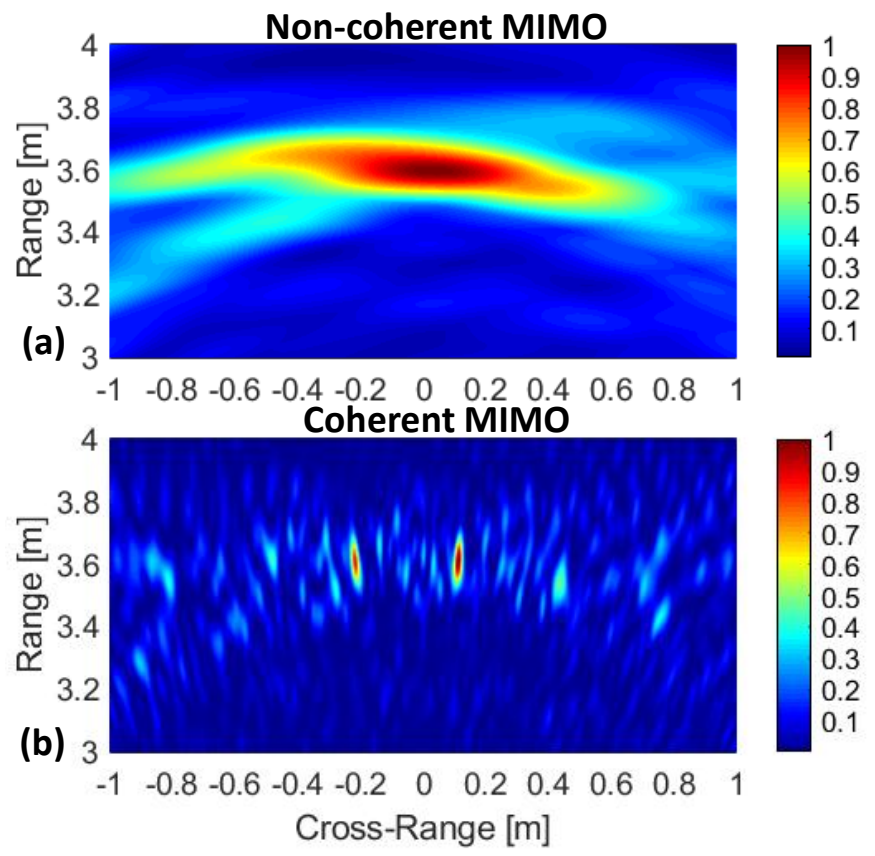

Fig. 5 Single-band 2x4 MIMO radar applied to two $30 \mathrm{~cm}$-spaced targets. (a) Non-coherent MIMO processing of real data, (b) coherent MIMO processing of real data. Experimental parameters: $f_{R F}=8.5 \mathrm{GHz}, B=1.4 \mathrm{GHz}$; Target positions $T_{1} \equiv(-0.2,+3.6) m ; T_{2} \equiv(0.1,+3.6) m$. may lead to possible amplitude mismatches between the different channels. This latter issue may be addressed by supplying each VCSEL with an adaptive gain controller. However, this problem goes out of the scope of our demonstration and it will be investigated in the future.

At last, a further example has been considered to highlight the superior capability of the coherent approach in distinguishing two closely spaced targets, which are identical to the one employed in the single-target case. As expected, since the relative spacing $-30 \mathrm{~cm}$ - in cross-range is less than half the cross-range resolution in case of non-coherent approach $62 \mathrm{~cm}$ - in this case a single extended lobe is obtained, see Fig. 5(a). On the contrary, when applying the coherent approach, the two targets are distinguishable with a minimal position error: target 1 , positioned at $(-0.2,+3.6) m$ is mapped at $(-0.215,+3.61) m$, while target 2 , positioned at $(+0.1,+3.6) m$ is mapped at $(+0.115,+3.61) m$, see Fig. 5(b). Residual sidelobes appear and the dense periodicity is most likely due to the interference of those relating to one target with those relating to the other.

\section{DUAL-BAND VCSEL-BASED RADAR NETWORK EXPERIMENT}

In this Section, both simulation and experimental results are presented and discussed, in order to compare the system performance in case of dual-band operation, with respect to the single-band case.

The experimental setup is the same as the one presented in Section III, with now a dual-band functionality (i.e., $L=2$ ). In particular, the following waveform parameters are considered: $f_{R F 1}=8 \mathrm{GHz}, \quad f_{R F 2}=9 \mathrm{GHz}, \quad B_{1}=B_{2}=400 \mathrm{MHz}$. This way, occupying the previous frequency band (i.e., with $f_{R F}=$ $8.5 \mathrm{GHz}$ and $B=1.4 \mathrm{GHz}$ ) with two $400 \mathrm{MHz}$ sub-bands $600 \mathrm{MHz}-$ spaced, the proposed dual-band system exploits only about $57 \%$ of the bandwidth employed in the single-band experiment reported before. In the proposed dual-band experiment, $\mathrm{TX}_{1}$ and $\mathrm{TX}_{2}$ transmit up- and down-sweep LFM$\mathrm{CW}$ signals, respectively. The signals are contemporaneously transmitted by $\mathrm{TX}_{1}$ and $\mathrm{TX}_{2}$, but they are separated in the frequency domain to limit mutual interferences and ensure an appropriate signal orthogonality. Specifically, when $\mathrm{TX}_{1}$ transmits at $f_{R F 1} \mathrm{TX}_{2}$ transmits at $f_{R F 2}$. At the successive waveform repetition interval, $\mathrm{TX}_{1}$ transmits at $f_{R F 2}$, instead, while $\mathrm{TX}_{2}$ transmits at $f_{R F 1}$. Then, the transmission scheduling restarts following this periodicity.

The behavior granted in case of dual-band operation with respect to single-band operation is shown in Fig. 6, with the main performance metrics being summarized in Table II. The non-coherent MIMO ambiguity function is calculated, according to Eq. (2), for the dual-band case, and shown in Fig. 6(a). As we can observe, the cross-range resolution $\triangle X R$ is significantly worse than the one achieved in Fig. 4(a) for the single-band case, respectively $1.98 \mathrm{~m}$ versus $0.62 \mathrm{~m}$. The reason must be found in the coarser range resolution $\Delta R$ due to the smaller bandwidth employed, in addition to the fact that the map shown by Fig. 6(a) is obtained by pure summation of the 
This is the author's version of an article that has been published in the IEEE Journal of Lightwave Technologies. Changes were made to this version by the publisher prior to publication. The final version of record is available at http://dx.doi.org/10.1109/JLT.2020.3011800

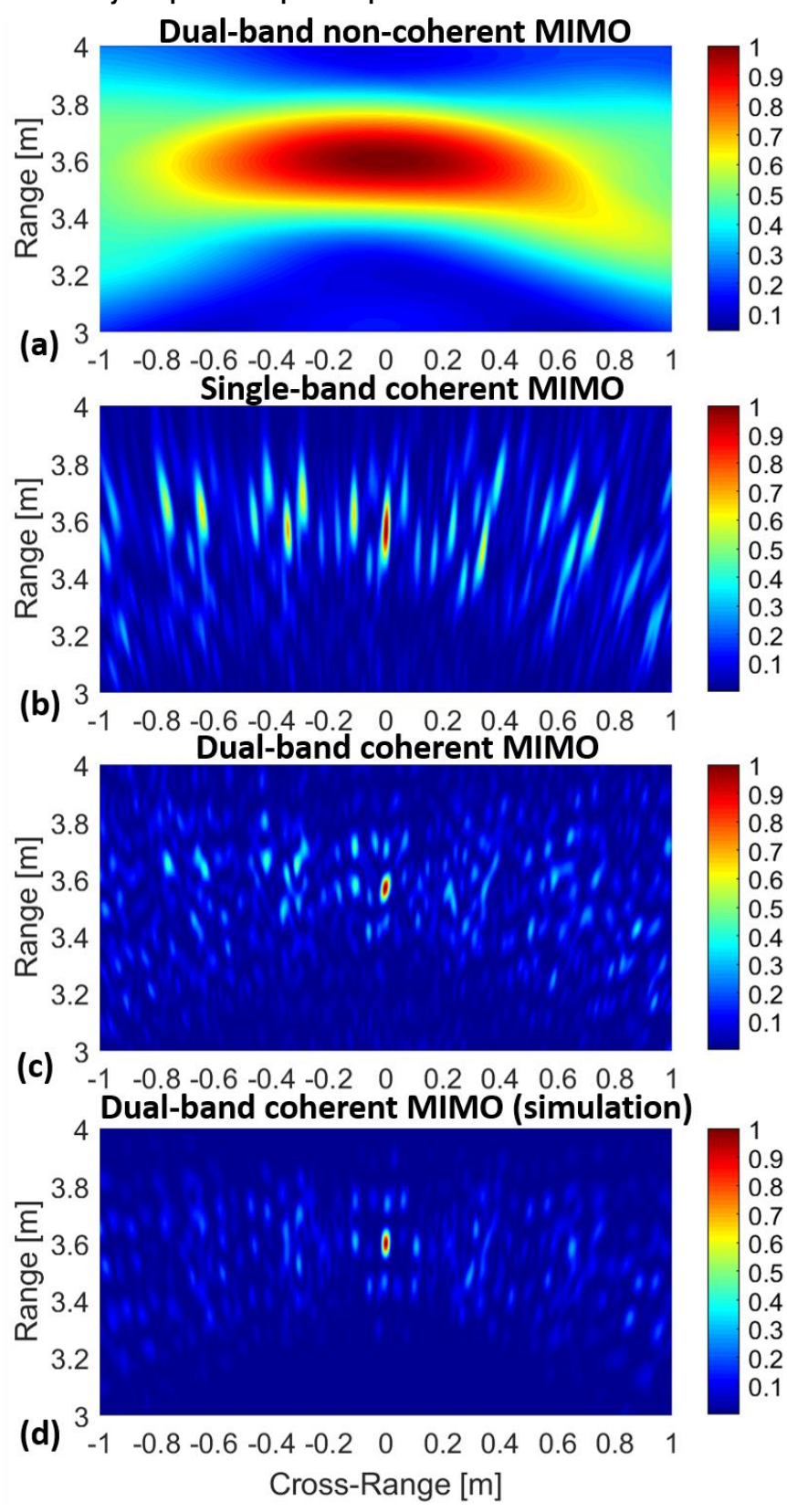

Fig. 6 Dual-band 2x4 MIMO radar experiment. Color-coded normalized MIMO ambiguity function in the range/cross-range Cartesian space for: (a) noncoherent MIMO processing of dual-band real data, (b) coherent MIMO processing of real data for the single-band configuration at $f_{R F 2}$, (c) coherent MIMO processing of dual-band real data, (d) coherent MIMO processing of dual-band simulated data. Experimental parameters: $f_{R F 1}=8 \mathrm{GHz}, f_{R F 2}=$ $9 \mathrm{GHz}, B 1=B 2=0.4 \mathrm{GHz}$; Target position $T \equiv(0,+3.6) \mathrm{m}$.

two single-band maps, corresponding to $B_{1}$ and $B_{2}$, respectively. Indeed, such intrinsically "non-coherent" operation does not narrow the resulting lobe with respect to each of the single-band cases. The coherent MIMO ambiguity function, which is calculated according to Eq. (4) for the singleband case at $f_{R F 2}=9 \mathrm{GHz}$, is shown in Fig. 6(b). Here, the target-originated peak can be clearly observed. However, many large sidelobes can also be spotted around the main peak. The high and numerous sidelobes are due to the limited MIMO array configuration, the geometry of the array with respect to the target, and the small fractional bandwidth $B_{2} / f_{R F 2}$. As a matter
TABLE II

\begin{tabular}{ccccc}
\multicolumn{4}{c}{ PERFORMANCE METRICS FOR THE DUAL-BAND EXPERIMENT } \\
\hline & \multicolumn{2}{c}{ Simulated Data } & \multicolumn{2}{c}{ Experimental Data } \\
& NC-MIMO & C-MIMO & NC-MIMO & C-MIMO \\
\hline$\Delta R$ & $0.39 \mathrm{~m}$ & $0.075 \mathrm{~m}$ & $0.39 \mathrm{~m}$ & $0.075 \mathrm{~m}$ \\
$\Delta X R$ & $1.9 \mathrm{~m}$ & $0.026 \mathrm{~m}$ & $1.98 \mathrm{~m}$ & $0.027 \mathrm{~m}$ \\
PMSR & - & $5.05 \mathrm{~dB}$ & - & $3.25 \mathrm{~dB}$ \\
PASR & - & $11.82 \mathrm{~dB}$ & - & $7.50 \mathrm{~dB}$ \\
\hline
\end{tabular}

NC/C-MIMO: Non-Coherent/Coherent MIMO; $\Delta R$ : Range Resolution; $\Delta X R$ : Cross-Range Resolution; PMSR/PASR: Peak-to-Maximum/AverageSidelobe-Ratio. Sidelobe statistics cannot be evaluated for NC-MIMO.

of fact, as highlighted in [8], this ratio must be at least 1/10 for a fruitful coherent MIMO processing.

Finally, the output of the dual-band coherent MIMO ambiguity function is shown in Fig. 6(c). As we can observe, the joint operation of signals at $f_{R F 1}$ and $f_{R F 2}$ is such that the secondary lobes are strongly mitigated with respect to the case in which the MIMO radar system operates at $f_{R F 1}$ or at $f_{R F 2}$ alone, see Fig. 6(b). Finally, the dual-band coherent MIMO ambiguity function obtained from the simulated data is depicted in Fig. 6 (d). As we can observe, the main difference with respect to the experimental result, consists of less pronounced spurious sidelobes, leading to a PASR of $11.82 \mathrm{~dB}$ whilst the experimental result exhibits $7.5 \mathrm{~dB}$. These differences may be ascribed to the same main reasons highlighted in Section III.B.

Additional simulation results, not included here, show how a similar $4 \times 8$ MIMO system achieves a PASR equal to 16 and $19 \mathrm{~dB}$, for the dual-band and single $1.4 \mathrm{GHz}$-band case, respectively. The estimated values of range $(\Delta R)$ and crossrange $(\triangle X R)$ resolutions, together with the PMSR and PASR, are reported for completeness in Table II.

Surprisingly, by comparing Tables I and II, even if only 57\% of bandwidth is employed in the dual-band case, a slightly better range resolution is obtained with respect to the single 1.4 GHz-band case, albeit at the expense of higher in-range sidelobes. Both phenomena are due to beating of the two employed carrier frequencies, when coherently summing the two ambiguity functions, in correspondence of the range area covered by the main lobe of each function. In this regard, an extensive analysis is required for defining a system design strategy for minimizing this effect in case of multi-band operation.

\section{SUMMARY AND CONCLUSION}

The potential of a radar network, where remoting of two transmitters and four receivers is achieved through optical fiber links based on directly modulated (DM) vertical-cavity surfaceemitting lasers (VCSELs), has been numerically and experimentally analyzed. Distribution of the radiofrequency (RF) signals through optical fibers, instead of intermediatefrequency (IF) ones, avoids up/down conversion at the remote radar frontends, which would prevent phase coherence among transmitted and received signals. This allows the so called coherent multiple-input multiple-output (MIMO) processing of raw signals which takes place at the centralized base station, permitting this way to reach superior range/cross-range resolution than through non-coherent data fusion.

However, since present VCSELs cannot handle RF carriers 
This is the author's version of an article that has been published in the IEEE Journal of Lightwave Technologies. Changes were made to this version by the publisher prior to publication. The final version of record is available at http://dx.doi.org/10.1109/JLT.2020.3011800

in excess to $30 \mathrm{GHz}$, in applications such as remote sensing for automotive where higher carriers are employed (e.g. $77 \mathrm{GHz}$ ), commercial integrated radar transceivers including frequency multipliers $(\times 4)$ and dividers $(\div 4)$ might be an option [31].

VCSEL-based signal distribution through optical links prevents electromagnetic interference issues, reduces the encumbrance of each link and is a low-cost and power efficient solution perfectly fitting on-board automotive scenarios. The employed $10 \mathrm{~m}$-long fiber spools enable TX/RX remoting on board of road vehicles and for indoor environment. In addition, the use of VCSELs with emission frequency in the $1.3 \mu \mathrm{m}$ regime avoids significant signal distortion induced by chromatic dispersion of standard fiber, enabling, in principle, TX/RX remoting up to kilometer reach, consequently making the presented solution attractive for other application areas too.

Coherent MIMO processing of $1.4 \mathrm{GHz}$-wide RF signals at $8.5 \mathrm{GHz}$-carrier frequency, from the $2 \times 4$ radar-over-fiber network sparse along a 3m-long baseline, successfully demonstrated cross-range resolution down to $2.5 \mathrm{~cm}$ at a $3.6 \mathrm{~m}$ distance. With the same hardware, coherent dual-band operation in the same RF range with two $400 \mathrm{MHz}$-wide bands with $600 \mathrm{MHz}$-vacant band in between, achieved cross-range resolution down to $2.7 \mathrm{~cm}$ at the same distance.

Authors believe the presented results clearly state that the proposed VCSEL-based signal distribution represents an effective way to enable coherent multi-band MIMO processing in a centralized radar network.

\section{ACKNOWLEDGMENT}

This work has been partially supported by Veoneer. Authors would like to thank Olof Eriksson and Oliver Brunnegard from Veoneer for technical support and Christian Neumeyr from Vertilas GmbH for having provided the TO-can VCSELs.

\section{REFERENCES}

[1] M. S. Greco, J. Li, T. Long and A. Zoubir, "Advances in Radar Systems for Modern Civilian and Commercial Applications: Part 1 [From the Guest Editors]," IEEE Signal Processing Magazine, vol. 36, no. 4, pp. 13-15, July 2019

[2] M. S. Greco, J. Li, T. Long and A. Zoubir, "Advances in Radar Systems for Modern Civilian and Commercial Applications: Part 2 [From the Guest Editors]," IEEE Signal Processing Magazine, vol. 36, no. 5, pp. 16-18, Sept. 2019

[3] J. J. Alter, R. M. White, F. F. Kretschmer, I. D. Olin and C. L. Temes, "Ubiquitous radar: an implementation concept," Proc. of the 2004 IEEE Radar Conf., Philadelphia, PA, USA, 2004, pp. 65-70

[4] Moreira, P. Prats-Iraola, M. Younis, G. Krieger, I. Hajnsek, K.P. Papathanassiou, "A tutorial on synthetic aperture radar," in IEEE Geoscience and Remote Sensing Magazine, vol. 1, no. 1, pp. 6-43, 2013.

[5] H. Godrich, A. M. Haimovich and R. S. Blum, "Target Localization Accuracy Gain in MIMO Radar-Based Systems," in IEEE Transactions on Information Theory, vol. 56, no. 6, pp. 2783-2803, June 2010.

[6] E. Fishler, A. Haimovich, R. Blum, D. Chizhik, L. Cimini and R. Valenzuela, "MIMO radar: an idea whose time has come," Proceedings of the 2004 IEEE Radar Conference (IEEE Cat. No.04CH37509), Philadelphia, PA, USA, 2004, pp. 71-78

[7] V. S. Chernyak, Fundamentals of Multisite Radar Systems. New York: Gordon and Breach, 1998

[8] A. M. Haimovich, R. S. Blum and L. J. Cimini, "MIMO Radar with Widely Separated Antennas," in IEEE Signal Processing Magazine, vol. 25, no. 1, pp. 116-129, 2008.

[9] N. H. Lehmann, A. M. Haimovich, R. S. Blum and L. Cimini, "High Resolution Capabilities of MIMO Radar," 2006 Fortieth Asilomar
Conference on Signals, Systems and Computers, Pacific Grove, CA, 2006, pp. 25-30.

[10] Chernyak, V. S. Fundamentals of Multisite Radar Systems: Multistatic Radars and Multistatic Radar Systems. 1st. s.1.: CRC Press, 1998.

[11] Hanle, E. Survey of bistatic and multistatic radar 1986, IEEE Proceedings on Communications, Radar and Signal Processing, Vol. 133, p. 587-595

[12] L. Lembo, P. Ghelfi, A. Bogoni, "Antenna Position Optimization in a MIMO Distributed Radar Network through Genetic Algorithms," 20th International Radar Symposium (IRS), Ulm, Germany, 2019, pp. 1-6.

[13] M. Vespe, C. J. Baker, and H. D. Griffiths, "Automatic target recognition using multi-diversity radar," IET Radar Sonar Navigation, vol. 1, no. 6, pp. 470-478, 2007.

[14] P. Lombardo, and D. Pastina, "Multiband coherent radar detection against compound-Gaussian clutter," IEEE Trans. Aerosp. Electron. Syst., vol. 35, no. 4, pp. 1266-1282, Oct. 1999.

[15] P. Van Dorp, R. Ebeling, and A. G. Huizing, "High resolution radar imaging using coherent multiband processing techniques," IEEE Radar Conf., Washington, DC, USA, 2010, pp. 981-986.

[16] X. Wei, Y. Zheng, Z. Cui, and Q. Wang, "Multi-band SAR images fusion using the EM algorithm in contourlet domain," Int. Fuzzy Syst. Knowledge Discovery Conf., Haikou, China, 2007, pp. 502-506.

[17] J. Li, and P. Stoica, "MIMO Radar Signal Processing," John Wiley \& Sons, Inc. Hoboken, New Jersey, USA, 2009

[18] F. Scotti, F. Laghezza, P. Ghelfi and A. Bogoni, "Multi-Band SoftwareDefined Coherent Radar Based on a Single Photonic Transceiver," IEEE Transactions on Microwave Theory and Techniques, vol. 63, no. 2, pp. 546-552, Feb. 2015

[19] K. M. Cuomo, J. E. Pion, and J. T. Mayhan, "Ultrawide-band coherent processing," IEEE Trans. Antennas Propag., vol. 47, no. 6, pp. 1094$1107,1999$.

[20] E. G. Larsson, P. Stoica and Jian Li, "Amplitude spectrum estimation for two-dimensional gapped data," in IEEE Transactions on Signal Processing, vol. 50, no. 6, pp. 1343-1354, June 2002.

[21] B. Hussain, A. Malacarne, S. Maresca, F. Scotti, P. Ghelfi and A. Bogoni, "Auto-regressive spectral gap filling algorithms for photonicsbased highly sparse coherent multi-band radars in complex scenarios," 2018 IEEE Radar Conference (RadarConf18), Oklahoma City, OK, 2018, pp. 0993-0998.

[22] G. Serafino et al., "Toward a New Generation of Radar Systems Based on Microwave Photonic Technologies," in Journal of Lightwave Technology, vol. 37, no. 2, pp. 643-650, 15 Jan.15, 2019.

[23] P. Ghelfi, F. Laghezza, F. Scotti, D. Onori and A. Bogoni, "Photonics for Radars Operating on Multiple Coherent Bands," in Journal of Lightwave Technology, vol. 34, no. 2, pp. 500-507, 15 Jan.15, 2016.

[24] A. Malacarne et al., "A Ultrawide-Band VCSEL-Based Radar-OverFiber System," 2019 International Topical Meeting on Microwave Photonics (MWP), Ottawa, ON, Canada, 2019, pp. 1-4.

[25] A. Malacarne, F. Scotti, S. Maresca, B. Hussain, L. Lembo, G. Serafino, A. Bogoni, P. Ghelfi, "A Radar-over-Fiber System based on DirectlyModulated Uncooled VCSELs", 45th European Conference on Optical Communication (ECOC 2019), paper P54, Dublin, 22-26 Sept. 2019

[26] M. Ortsiefer et al., "Long wavelength high speed VCSELs for long haul and data centers," OFC 2014, San Francisco, CA, 2014, pp. 1-3.

[27] T. Aalto et al., "Transceivers for $400 \mathrm{G}$ based on Hybrid Integrated Thick OI and III/V Chips," 2017 European Conference on Optical Communication (ECOC), Gothenburg, 2017, pp. 1-3.

[28] A. Malacarne et al., "Optical Transmitter Based on a 1.3- $\mu \mathrm{m}$ VCSEL and a SiGe Driver Circuit for Short-Reach Applications and Beyond," in Journal of Lightwave Technology, vol. 36, no. 9, pp. 1527-1536, 1 May1, 2018.

[29] D. M. Kuchta et al., "A 71-Gb/s NRZ Modulated 850-nm VCSEL-Based Optical Link," in IEEE Photonics Technology Letters, vol. 27, no. 6, pp. 577-580, 15 March15, 2015.

[30] W. Hofmann et al., "40 Gbit/s modulation of $1550 \mathrm{~nm} \mathrm{VCSEL,"} \mathrm{in}$ Electronics Letters, vol. 47, no. 4, pp. 270-271, 17 February 2011.

[31] S. Preussler, F. Schwartau, J. Schoebel, and T. Schneider, "Photonically synchronized large aperture radar for autonomous driving," OSA Optics Express, vol. 27, no. 2, pp. 1199-1207, 2019.

[32] L. Lembo, P. Ghelfi and A. Bogoni, "Analysis of a Coherent Distributed MIMO Photonics-Based Radar Network," 2018 15th European Radar Conference (EuRAD), Madrid, 2018, pp. 170-173

[33] S. Maresca et al., "Photonics for Coherent MIMO Radar: an Experimental Multi-Target Surveillance Scenario," 2019 20th International Radar Symposium (IRS), Ulm, Germany, 2019, pp. 1-6. 